\title{
Quantitation of CDC6 and MCM5 mRNA in cervical intraepithelial neoplasia and invasive squamous cell carcinoma of the cervix
}

\author{
Niamh Murphy ${ }^{1,3}$, Martina Ring ${ }^{1,3}$, Cynthia CBB Heffron ${ }^{1,3}$, Cara M Martin ${ }^{1,3}$, \\ Eamon McGuinness $^{4}$, Orla Sheils ${ }^{2,3}$ and John J O’Leary ${ }^{1,2,3}$ \\ ${ }^{1}$ Department of Pathology, Coombe Women's Hospital, Dublin, Ireland; ${ }^{2}$ Department of Histopathology, \\ St James Hospital, Dublin, Ireland; ${ }^{3}$ Trinity College Dublin, Dublin, Ireland and ${ }^{4}$ Department of Obstetrics \\ and Gynaecology, Coombe Women's Hospital, Dublin, Ireland
}

\begin{abstract}
CDC6 and MCM5 play essential roles in eukaryotic DNA replication. Several studies have highlighted the potential of these proteins as molecular markers of dysplastic and malignant cells in histopathological diagnosis. The mode of expression of CDC6 and MCM5 MRNA and their significance in normal, dysplastic and malignant cervical cells remains to be elucidated. Using a quantitative real-time RT PCR assay, we compared CDC6 and MCM5 mRNA expression in normal cervical epithelium, cervical intraepithelial neoplasia and invasive squamous cell carcinoma of the cervix. Our study cohort comprised 20 normal cervical biopsies, 20 CIN3 and eight invasive squamous cell carcinomas. All samples were formalin fixed and paraffin embedded. Total RNA was extracted and analysed for expression of GAPDH, CDC6 and MCM5 using real-time quantitative TaqMan RTPCR. A linear increase in MCM5 and CDC6 mRNA expression is observed in normal cervix, CIN3 and invasive cervical carcinoma. The overall difference in MCM5 mRNA expression in the normal cervix, CIN3 and invasive cohort groups is highly statistically significant $(P=0.001)$. An increase in CDC6 mRNA expression in CIN3 and invasive cervical squamous cell carcinoma was observed; however, the overall difference between cohort groups was not found to be statistically significant $(P=0.104)$. Increased transcription of MCM5 and CDC6 occurs as a consequence of cervical neoplastic progression. This pattern of increased mRNA expression in CIN3 and invasive cervical carcinoma directly correlates with findings at the phenotypic protein expression level. This study further confirms the importance of MCM5 and CDC6 in malignant transformation and in the pathogenesis of cervical dysplasia.

Modern Pathology (2005) 18, 844-849, advance online publication, 4 February 2005; doi:10.1038/modpathol.3800361
\end{abstract}

Keywords: CDC6; MCM5; CIN; quantitative Taqman RT-PCR

In all eukaryotes, a conserved mechanism of DNA replication exists, which ensures that DNA replication occurs only once in a single cell cycle. ${ }^{1}$ This mechanism is often termed the 'licensing' of DNA replication. ${ }^{2}$ DNA replication requires the regulated assembly of pre-replicative complexes (pre-RC) onto DNA during the G1 phase of the cell cycle. Pre-RCs render the chromatin competent or 'licensed' to replicate. Among the proteins known to assemble to form the pre-RC are cell division cycle protein 6 (CDC6) and mini chromosome maintenance (MCM) proteins. $^{3,4}$

Correspondence: Professor JJ O’Leary, Department of Pathology, Coombe Women's Hospital, Dublin 8, Ireland.

E-mail: olearyjj@tcd.ie

Received 28 September 2004; revised and accepted 4 November 2004; published online 4 February 2005
Biological analysis of CDC6 suggests that it functions as a clamp loader in which ATP binding and hydrolysis induce conformational changes, which result in the recruitment or loading of MCMs onto DNA. ${ }^{3}$ Once the MCMs are recruited to the preRC, CDC6 protein is then phosphorylated by Cyclin $\mathrm{A} / \mathrm{CDK} 2$ in $\mathrm{S}$ phase of the cell cycle. This results in the translocation of CDC6 from its chromatin sites to the cytoplasm where it is degraded by the anaphase promoting complex (APC)/cyclosome. ${ }^{5-8}$ Relocalisation of CDC6 to the cytoplasm prevents reinitiation of replication. In addition to conferring replication competence, studies also suggest that the level/ modification of CDC6 in G2 phase functions as a checkpoint control, which ensures that the $\mathrm{S}$ phase nucleus has completed replication before entry in mitosis. ${ }^{9-11}$

The eukaryotic MCM is a family of six essential proteins (MCM2-7) all of which are essential for 
replication fork progression. ${ }^{3}$ The MCM proteins form a heterohexameric ring-shaped complex, which has helicase activity. Laskey et al, 2003 proposed that the MCM hexameric ring acts as a rotary motor, which pumps DNA along its helicase axis, by simple rotation in a manner comparable to the movement of a threaded bolt through a nut. Firstly, in G1 phase the MCM hexameric complexes are loaded onto DNA at the origin of replication. The MCM ring complexes then move away from the origin by rotation along the helical thread of DNA. In the second part of this model, in S phase the now dispersed MCM ring complexes become immobilised. The same rotary action is repeated but now only the DNA is capable of rotation. ${ }^{12}$ This action results in pumping the DNA back towards replication forks and also results in its unwinding at the replication fork. As replication precedes, the length of unreplicated DNA shortens and the MCM complex becomes dissociated from DNA. ${ }^{12,13}$

Deregulation of DNA replication results in genomic instability and contributes to the malignant transformation of cells. ${ }^{2,14}$ Not surprisingly then, changes in the expression pattern of DNA 'licensing' proteins are frequently observed in dysplastic cells. In normal cells, MCM5 and CDC6 are present only during the cell cycle and are lost from the cell during quiescence and differentiation. ${ }^{15}$ As a consequence, MCM5 and CDC6 have been proposed as specific biomarkers of proliferating cells. Dysplastic cells are characterised in functional terms as remaining in the cell cycle and numerous studies have reported elevated levels of MCM5 and CDC6 protein expression in dysplastic cells. A number of published studies have highlighted their potential use in the identification and/or diagnosis of a range of dysplastic and neoplastic conditions. Davidson et $a l,{ }^{16}$ reported that MCM5 identified all cells actively involved in the cell cycle in vulval intraepithelial neoplastic lesions (VIN) and proposed that immunostaining of VIN lesions with antibodies against MCM proteins might have a number of clinical applications. Other studies have highlighted the use of aberrant overexpression of MCM proteins as a marker of proliferation potential in dysplastic squamous oesophageal epithelium and Barrett's mucosa. ${ }^{17} \mathrm{~A}$ study by Stober et $a l^{15}$ demonstrated that immunofluorometric detection of MCM5 in urine sediments could be employed as a sensitive and specific diagnostic test for bladder cancer. Detection of CDC6 protein may also have clinical relevance, for example, CDC6 protein can be used for the estimation of proliferative activity of brain tumours. ${ }^{18}$

Increased expression of CDC6 and MCM5 proteins has been demonstrated using immunohistochemical detection methods in preinvasive and invasive cells of the cervix. ${ }^{14,19-22}$ This aberrant overexpression of MCM5 and CDC6 has been suggested as a potential molecular marker of cervical squamous and glandular neoplasia both in biopsy sections and in cytology smears. ${ }^{19-22}$ The mRNA expression profile of CDC6 and MCM5 and its significance in normal, dysplastic and malignant cervical cells remains to be elucidated. The aim of this study was to examine and compare the mRNA expression profiles of MCM5 and CDC6 in the normal cervix, preinvasive lesions and invasive carcinomas of the cervix using quantitative real-time PCR assays.

\section{Materials and methods}

\section{Tissue Samples}

MCM5 and CDC6 mRNA expression was examined in 20 normal cervical biopsies, 20 cervical intraepithelial neoplasia grade 3 (CIN3) biopsies and eight invasive cervical squamous cell carcinomas. The CIN3 cohort was selected as being representative of dysplasia. All material was fixed in $10 \%$ buffered formal saline and embedded in paraffin wax. Diagnosis was confirmed on H\&E-stained sections for all biopsy samples by a histopathologist according to the criteria outlined by the World Health Organisation.

\section{mRNA Extraction}

RNA extraction from formalin-fixed paraffin-embedded archival material was carried out using the Gentra Systems Purescript RNA Isolation Kit (Puregene, Minneapolis). With reference to $\mathrm{H} \& \mathrm{E}$-stained sections, the appropriate area of tissue/tumour was selected macroscopically. The selected area from the slide was then matched to the corresponding paraffin-embedded block. The perimeter of the selected area was scored with a sterile scalpel. Four $(10 \mu \mathrm{m})$ sections of the selected area were cut using a microtome and placed in a sterile $1.5 \mathrm{ml}$ microcentrifuge tube. The extracted RNA was used as template in subsequent GAPDH, MCM5 and CDC6 One Step RT-PCR TaqMan reactions.

\section{CDC6 and MCM5 TaqMan RT-PCR}

Real-time quantitative RT-PCR based on the $5^{\prime}$ nuclease assay was performed on an ABI 7000 sequence detector (Applied Biosystems). Sequencespecific PCR primers and TaqMan MGB probes were designed using Primer Express Software versions 1.5-1.7 (Applied Biosystems, Lincoln Centre drive, Foster City, CA, USA) (Table 1). All quantitative PCRs were prepared in a dedicated facility in a class 2 laminar flow benchhood using dedicated pipettors and aerosol-resistant pipette tips.

Following optimisation, quantitative TaqMan RTPCR reactions for MCM5 and CDC6 were performed in duplicate under the following conditions for each $25 \mu \mathrm{l}$ reaction: $1 \times$ One-Step RT-PCR Mastermix, $100 \mathrm{nM}$ probe, $200 \mathrm{nM}$ forward and reverse primers and $\approx 50$ ng RNA. Thermal cycling conditions were 
Table 1 Primer and probe sequences for MCM5 and CDC6

\begin{tabular}{ll}
\hline Name & Sequence $5^{\prime}-3^{\prime}$ \\
\hline $\begin{array}{l}\text { MCM5 TaqMan forward } \\
\text { primer }\end{array}$ & GGTCCTCCTGGCTGGTGAA \\
$\begin{array}{l}\text { MCM5 TaqMan reverse } \\
\text { primer }\end{array}$ & ACGTTGGATGCTGCCTTGTC \\
$\begin{array}{l}\text { MCM5 TaqMan MGB } \\
\text { probe }\end{array}$ & CCTCCACCCCTGACAG \\
$\begin{array}{l}\text { CDC6 TaqMan forward } \\
\text { primer }\end{array}$ & CTTTGGTGGAGAACAAGGAGGTAA \\
$\begin{array}{l}\text { CDC6 TaqMan reverse } \\
\text { CDimer }\end{array}$ & GTGTAAAAGCCCTGCCTCTCAG \\
\hline
\end{tabular}

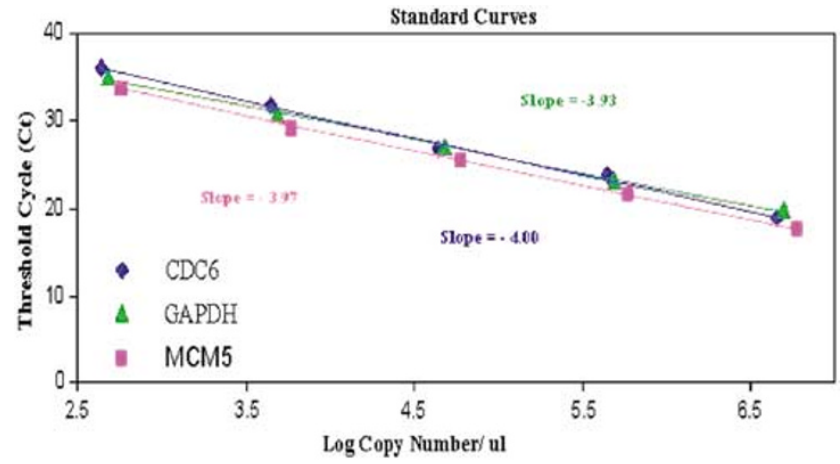

Figure 1 MCM5, CDC6 and GAPDH standard curves. A TaqMan RT-PCR standard curve for GAPDH was generated by amplifying serial dilutions of control human RNA (Applied Biosystems). MCM5 and CDC6 were produced by amplifying serial dilutions of cRNA standards $\left(10^{6}-10^{2}\right.$ copies $)$.

as follows: $2 \mathrm{~min}$ at $50^{\circ} \mathrm{C}, 30 \mathrm{~min}$ at $48^{\circ} \mathrm{C}, 10 \mathrm{~min}$ at $95^{\circ} \mathrm{C}$ and 40 cycles of $15 \mathrm{~s}$ at $95^{\circ} \mathrm{C}$ and $1 \mathrm{~min}$ at $60^{\circ} \mathrm{C}$. A total of six negatives (no template controls) were included in each TaqMan run.

To demonstrate the linearity and efficiency of the reverse transcription and TaqMan reactions and for the purpose of quantification, standard curves were generated. A gene dosage correction was carried using glyceraldehyde phosphate dehydrogenase as a housekeeping gene. A standard curve of GAPDH was generated by performing a serial dilution on control human RNA (Applied Biosystems) and amplifying as per test samples. MCM5 and CDC6 quantitative TaqMan RT-PCR was performed by generating standard curves for the MCM5 and CDC6 genes (Figure 1). TaqMan RT-PCR standards were generated by cloning MCM5 and CDC6 genespecific PCR products into a vector using the TOPO TA Cloning ${ }^{\circledR}$ system (Invitrogen, Groningen, the Netherlands) according to the manufacturer's instructions. Plasmids containing the PCR inserts were then in vitro transcribed into cDNA using the RiboProbe $^{\circledR}$ Combination system-SP6/T7 RNAPolymerase (Promega Corporation, WI, USA). Standard curves were generated by amplifying serial dilutions $\left(10^{6}-10^{2}\right.$ copies) of cRNA standards.

\section{Analysis of Results}

A normalised MCM5/CDC6 value or expression index for each experimental sample was calculated by dividing the MCM5/CDC6 copy number (determined from the appropriate standard curve) by the GAPDH (endogenous reference) copy number. Statistical analysis of MCM5 and CDC6 RT-PCR TaqMan results was carried out using SPSS v11 Software. The significance of different levels of MCM5 and CDC6 mRNA expression in normal CIN and invasive groups was assessed using the Kruskal-Wallis test. The Mann-Whitney $U$-test was used to assess pairwise differences between the two cohort groups. The relationship between mRNA expression and increasing severity of dysplasia was assessed using simple linear regression analysis.

\section{Results}

\section{MCM5 Quantitative Taqman RT-PCR}

The expression of MCM5, CDC6 and GAPDH mRNA was examined in 20 normal cervical biopsies, 20 CIN3 biopsies and eight invasive cervical squamous cell carcinomas. The normalised value or expression index for each experimental sample was determined. Figure 2 illustrates the increase in MCM5 mRNA expression levels in CIN3 and in invasive squamous carcinoma. When assessed using the Kruskal-Wallis test, the overall difference of MCM5 mRNA expression in the normal cervix, CIN3 and invasive cohort groups is highly statistically significant $(P=0.001)$. In addition, when the Mann-Whitney $U$-test was employed to assess pairwise differences between groups, a statistically

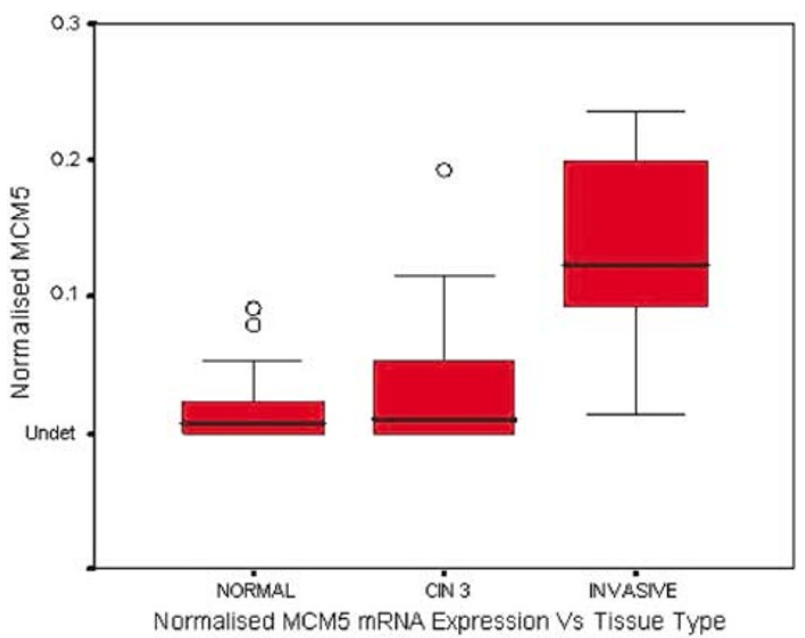

Figure 2 Box and Whisker plots of MCM5 mRNA expression in normal cervical biopsies, CIN3 lesions and invasive squamous cell carcinoma. The boxes depict the upper and lower quartiles. The 'whiskers' show the range with outliers marked by small circles. 
significant difference was observed between the normal and CIN3 groups $(P=0.001)$ as well as the normal and invasive groups $(P=0.000)$. While a difference in mRNA expression was observed between the CIN3 and invasive groups, this difference was not found to be statistically significant using the Mann-Whitney $U$-test $(P=0.512)$. Simple linear regression analysis revealed a highly significant linear relationship (estimated slope $=6.8$, $R^{2}=0.3, \quad P$-value $<0.000$ ) between the level of MCM5 mRNA expression in normal, CIN3 and invasive carcinoma.

\section{CDC6 Quantitative TaqMan RT-PCR}

The results of CDC6 mRNA quantitation are summarised in Figure 3. Although an increase in CDC6 mRNA expression levels in CIN3 and invasive cervical squamous cell carcinoma is evident from the graph, the increase is significantly less than that observed for MCM5. When assessed using the Kruskal-Wallis test, the overall difference in CDC6 mRNA expression in the normal cervix, CIN3 and invasive cohort groups was not found to be statistically significant $(P=0.104)$. In addition, when the Mann-Whitney $U$-test was employed to assess pairwise differences between groups, a statistically significant difference was not observed between the normal and CIN3 groups $(P=0.314)$ or the CIN3 and invasive groups $(P=0.281)$. However, a statistically significant difference in mRNA expression was observed between the normal and invasive groups $(P=0.050)$. Simple linear regression analysis revealed a linear relationship (estimated slope $=12.1, R^{2}=0.085, P$-value $<0.045$ ) between the level of CDC6 mRNA expression in normal

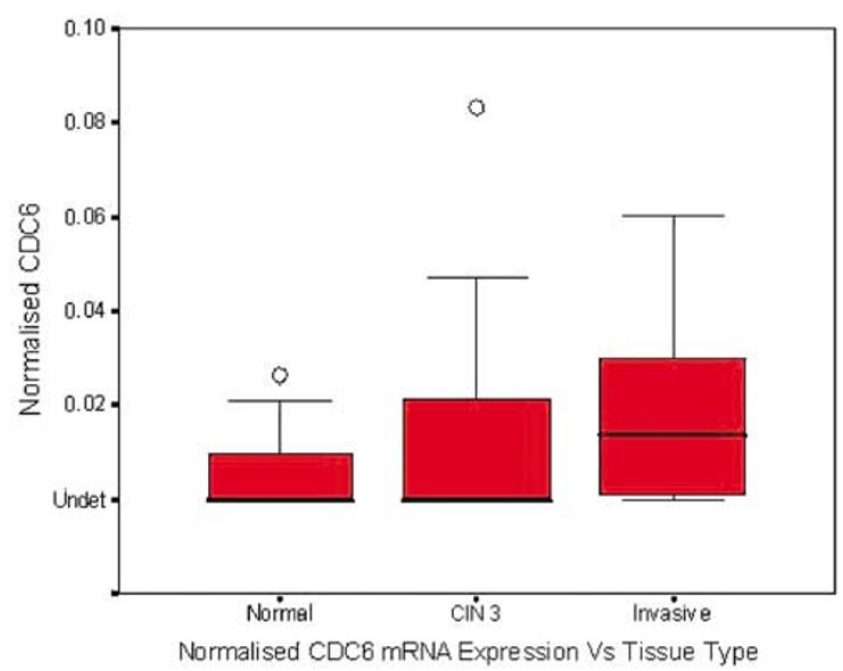

Figure 3 Box and Whisker plots of CDC6 mRNA expression in normal cervical biopsies, CIN 3 lesions and invasive squamous cell carcinoma. The boxes depict the upper and lower quartiles. The 'whiskers' show the range with outliers marked by small circles. cervix, CIN3 and invasive carcinoma. These results demonstrate that the increase in the level of expression of CDC6 mRNA in CIN3 and invasive carcinoma is considerably lower than that observed for MCM5. These results at the mRNA level correlate strongly with previous findings for CDC6 phenotypic protein expression.

\section{Discussion}

MCM5 and CDC6 play essential roles in the regulation of eukaryotic DNA replication. Both MCM5 and CDC6 protein are detectable in proliferating cells by immunohistochemical analysis but are not expressed in quiescent or differentiated cells. As a consequence, MCM5 and CDC6 appear to act as specific markers of cellular proliferation. ${ }^{22}$ A number of studies including our own have demonstrated increased MCM5 and CDC6 protein expression in neoplastic cervical cells and have highlighted their potential use in the diagnosis of cervical dysplasia. ${ }^{19-22}$ While MCM5 and CDC6 expression have been well characterised at the protein level, the mRNA expression profiles of CDC6 and MCM5 mRNA and their significance in normal cervix, preinvasive neoplastic and malignant cervical cells remain to be elucidated. In this study, quantitative real-time TaqMan RT-PCR was employed to elucidate the mRNA expression profile of MCM5 and CDC6 in normal cervix, preinvasive neoplastic and malignant cervical cells of the cervix.

Immunohistochemical analysis has demonstrated that MCM5 protein expression in the normal cervical epithelium is strictly confined to the basal proliferating layer. Increased MCM5 protein expression is detectable in all grades of glandular and squamous cervical dysplasia and this increase is proportional to the severity of dysplasia. ${ }^{14,19-22}$ This study has for the first time examined MCM5 mRNA expression in normal and dysplastic squamous cells of the cervix using quantitative TaqMan RTPCR. In this study, a linear increase in MCM5 mRNA expression was observed in the normal cervix, CIN3 and invasive carcinoma (estimated slope $=6.8$, $R^{2}=0.3, P$-value $\left.<0.000\right)$. Importantly, these results demonstrate an increase in MCM5 mRNA expression with increasing severity of dysplasia. Indeed, MCM5 mRNA expression closely correlates with findings at the phenotypic protein expression level, which demonstrated an increase in MCM5 protein expression with increasing severity of dysplasia, using immunohistochemical techniques. ${ }^{19}$ These findings suggest that increased MCM5 protein expression in cervical dysplasia is a consequence of transcriptional upregulation. One possible suggestion for upregulation of MCM5 mRNA in cervical dysplasia put forward by Ishimi et a ${ }^{14}$ could be that high-risk human papillomavirus oncoprotein E7 binding to the retinoblastoma protein releases inhibition on E2F allowing transcriptional upregu- 
lation of MCM5. However, the observed increase in MCM5 protein expression in a variety of non-HPVassociated neoplasms indicates that while increased expression of MCM5 in cervical dysplastic lesions may be associated with HPV infection it is not dependent on it. Indeed, it is likely that transcriptional upregulation of MCM5 may occur by a variety of mechanisms, which allow the release of E2F transcriptional inhibition, and it is likely that the exact mechanism may be tumour-type dependent. As a consequence, MCM5 may have a role as a biomarker of both HPV-dependent and -independent cervical neoplasia.

CDC6 protein expression has been shown by immunohistochemical analysis to be absent or confined to the basal proliferating layer in the normal cervical epithelium, while increased CDC6 protein expression is detected in all grades of cervical squamous and glandular dysplasia. CDC6 appears to be preferentially unregulated in highgrade preinvasive neoplasia (CIN3) and in invasive cervical carcinoma and in adenocarcinoma. ${ }^{19,20}$ In this study, simple linear regression analysis revealed a linear increase (estimated slope $=12.1$, $R^{2}=0.085, P$-value $\left.<0.045\right)$ in the level of CDC6 mRNA expression in the normal cervix, CIN3 and invasive carcinoma. These results at the mRNA level correlate strongly with previous findings for CDC6 phenotypic protein expression using immunohistochemical analysis, which demonstrated a linear relationship between increasing CDC6 protein expression and increasing severity of cervical dysplasia. Increased CDC6 mRNA expression in cervical neoplasia may be a consequence of high-risk human papillomavirus oncoprotein E7 binding to the retinoblastoma protein, resulting in the release of inhibition on E2F allowing transcriptional upregulation of the CDC6 gene. In a manner similar to MCM5, these results indicate that increased CDC6 protein expression is a consequence of transcriptional upregulation.

Interestingly, in this study we observed that the overall increase in CDC6 mRNA expression in dysplastic cells was markedly lower than that observed for MCM5 mRNA expression. This is not a consequence of disparity in assay efficiency as the slope of both assay standard curves, which is indicative of assay efficiency, is close to equal. In a previous study carried out by our group, it was observed that the overall increase in MCM5 protein expression in cervical dysplasia was higher than that observed for CDC6 protein expression. ${ }^{19}$ This correlates with findings at the mRNA level in the current study. The lower mRNA expression of CDC6 in cervical dysplasia may be a function of its role as a G2/M phase checkpoint regulator. Degradation of CDC6 is necessary for entry into the $M$ phase of the cell cycle. ${ }^{23}$ This may explain why CDC6 is expressed at relatively lower levels in neoplastic cells when compared with MCM5 mRNA expression. It is possible that as a consequence of neoplastic progression, high-grade neoplastic and malignant cells may find a mechanism to evade CDC6 G2/M phase regulation and thus continue proliferating in the presence of elevated levels of CDC6 protein.

In conclusion, this study illustrates an increase in MCM5 and CDC6 mRNA expression in cervical preinvasive neoplasia and invasive cervical carcinoma. This directly correlates with findings at the phenotypic protein expression level and indicates that increased MCM5 and CDC6 transcription in cervical dysplasia is a consequence of neoplastic progression. This study confirms the importance of MCM5 and CDC6 in malignant transformation and in the pathogenesis of cervical dysplasia.

\section{Acknowledgements}

We greatly acknowledge the support of the Royal City of Dublin, Hospital Trust, the Irish Cancer Society and the Health Research Board for funding this study.

\section{References}

1 Diffley JF, Labib K. The chromosome replication cycle. J Cell Sci 2002;115:869-872.

2 Takisawa H, Mimura S, Kubota Y. Eukaryotic DNA replication: from pre-replication complex to initiation complex. Curr Opin Cell Biol 2000;12:690-696.

3 Shin JH, Grabowski B, Kasiviswanathan R, et al. Regulation of minichromosome maintenance helicase activity by Cdc6. J Biol Chem 2003;278:38059-38067.

4 Cook JG, Park CH, Burke TW, et al. Analysis of Cdc6 function in the assembly of mammalian prereplication complexes. Proc Natl Acad Sci USA 2002;99: 1347-1352.

5 Biermann E, Baack M, Kreitz S, et al. Synthesis and turnover of the replicative CDC6 protein during the HeLa cell cycle. Eur J Biochem 2002;269:1040-1046.

6 Petersen BO, Wagener C, Marinoni F, et al. Cell cycleand cell growth-regulated proteolysis of mammalian CDC6 is dependent on APC-CDH1. Genes Dev 2000;14: 2330-2343.

7 Petersen BO, Lukas J, Sorensen C, et al. Phosphorylation of mammalian Cdc6 by Cyclin A/CDK2 regulates its subcellular localization. EMBO J 1999;18:396-410.

8 Saha P, Chen J, Lawlis SJ, et al. Human CDC6/Cdc18 associates with Orc1 and cyclin-cdk and is selectively eliminated from the nucleus at the onset of $\mathrm{S}$ phase. Mol Cell Biol 1998;18:2758-2767.

9 Clay-Farrace L, Pelizon C, Santamaria D, et al. Human replication protein Cdc6 prevents mitosis through a mechanism that implicates Chk1. EMBO J 2003;22: 704-712.

10 Yanow SK, Lygerou Z, Nurse P. Expression of Cdc18/ Cdc6 and Cdt1 during G2 phase induces initiation of DNA replication. EMBO J 2001;20:4648-4656.

11 Coverley D, Pelizon C, Trewick S, et al. Chromatinbound Cdc6 persists in S and G2 phases in human cells, while soluble Cdc6 is destroyed in a cyclin 
A-cdk2 dependent process. J Cell Sci 2000;113: 1929-1938.

12 Laskey R, Madine MA. A rotary pumping model for helicase function of MCM proteins at a distance from replication forks. EMBO Rep 2003;4:26-30.

13 Shechter D, Ying CY, Gautier J. DNA unwinding is a MCM-complex and ATP-hydrolysis dependent process. J Biol Chem 2004;279:45586-45593.

14 Ishimi Y, Okayasu I, Kato C, et al. Enhanced expression of MCM proteins in cells derived from the uterine cervix. Eur J Biochem 2003;270:1089-1101.

15 Stoeber K, Swinn R, Prevost AT, et al. Diagnosis of genito-urinary tract cancer by detection of minichromosome maintenance 5 protein in urine sediments. J Natl Cancer Inst 2002;94:1071-1079.

16 Davidson EJ, Morris LS, Scott IS, et al. Minichromosome maintenance (Mcm) proteins, cyclin B1 and D1, phosphohistone $\mathrm{H} 3$ and in situ DNA replication for functional analysis of vulval intraepithelial neoplasia. Br J Cancer 2003;88:257-262.

17 Going JJ, Keith WN, Neilson L, et al. Aberrant expression of minichromosome maintenance proteins 2 and 5, and Ki-67 in dysplastic squamous oeso- phageal epithelium and Barrett's mucosa. Gut 2002;50: 373-377.

18 Ohta S, Koide M, Tokuyama T, et al. Cdc6 expression as a marker of proliferative activity in brain tumors. Oncol Rep 2001;8:1063-1066.

19 Muphy N, Ring M, Heffron CCBB, et al. p16 $6^{\mathrm{INK} 4 \mathrm{~A}}$, CDC6 and MCM5: predictive biomarkers in cervical preinvasive neoplasia and cervical cancer, in press.

20 Bonds L, Baker P, Gup C, et al. Immunohistochemical localization of CDC6 in Squamous and Glandular Neoplasia of the Uterine Cervix. Arch Pathol Lab Med 2002;126:1164-1168.

21 Freeman A, Morris LS, Mills AD, et al. Minichromosome maintenance proteins as biological markers of dysplasia and malignancy. Clin Cancer Res 1999;5: 2121-2132.

22 Williams GH, Romanowski P, Morris L, et al. Improved cervical smear assessment using antibodies against proteins that regulate DNA replication. Proc Natl Acad Sci USA 1998;95:14932-14937.

23 Robles L, Frost A, Davila M, et al. Down-regulation of Cdc6, a Cell Cycle Regulatory Gene, in Prostate Cancer. J Biol Chem 2002;277:25431-25438. 